\title{
Revisiting a null hypothesis: exploring the parameters of oligometastasis treatment
}

\author{
Jessica A. Scaborough ${ }^{1,2}$, Martin C. Tom ${ }^{3}$, Michael W. Kattan ${ }^{4}$, and Jacob G. Scott ${ }^{1,2,5 凶}$ \\ ${ }^{1}$ Translational Hematology and Oncology Research, Cleveland Clinic \\ ${ }^{2}$ Systems Biology and Bioinformatics Program, Department of Nutrition, Case Western Reserve School of Medicine \\ ${ }^{3}$ Department of Radiation Oncology, Miami Cancer Institute, Baptist Health South Florida, Miami, Florida \\ ${ }^{4}$ Department of Quantitative Health Sciences, Cleveland Clinic \\ ${ }^{5}$ Radiation Oncology, Cleveland Clinic
}

\begin{abstract}
In the treatment of patients with metastatic cancer, the cur- 15 rent paradigm states that metastasis-directed therapy does not ${ }_{16}$ prolong life. This paradigm forms the basis of clinical trial null hypotheses, where trials are built to test the null hypothesis: patients garner no overall survival benefit from targeting metastatic lesions. However, with advancing imaging technology and increasingly precise techniques for targeting lesions, a much larger proportion of metastatic disease can be treated. As ${ }^{2}$ a result, the life-extending benefit of targeting metastatic dis- 22 ease is becoming increasingly clear. In this work, we suggest 23 shifting this qualitative null hypothesis, and describe a mathe- 24 matical model which can be used to frame a new, quantitative ${ }_{25}$ null. We begin with a very simple formulation of tumor growth, 26 an exponential function, and illustrate how the same intervention (removing a given number of cells from the tumor) at different times effects survival. Additionally, we postulate where recent clinical trials fit into this parameter space and discuss the implications of clinical trial design in changing these quantitative parameters. Our model shows that while any amount of cell kill will extend survival, in many cases the extent is so small as to be unnoticeable in a clinical context or out-weighed by factors related to toxicity and treatment time. Recasting the null in these quantitative terms will allow trialists to design trials specifically to increase understanding of what circumstances (patient selection, disease burden, tumor growth kinetics) can lead to improved OS when targeting metastatic lesions, rather than whether or not targeting metastases extends survival for patients with (oligo-)metastatic disease.
\end{abstract}

oligometastasis | mathematical oncology | radiation therapy | cancer Correspondence: scottj10@ccf.org

\section{Introduction}

In the treatment of patients with metastatic cancer, the cur- 46 rent paradigm states that targeted treatment of metastatic le- 47 sions does not prolong life. This paradigm forms the basis 48 of clinical trial null hypotheses, where trials are built to test 49 the null hypothesis: patients garner no overall survival (OS) 50 benefit from targeting metastatic lesions.

51

The development of distant metastases is the forerunner of 52 cancer-related death (1-3). A Hallmark of Cancer, the dis- 53 semination of cancer cells from their origin to distant sites 54 results from a complex cascade of biological events, which 55 may subsequently allow for even more efficient tumor propa- 56 gation (4-6). Eradicating the body of as much metastatic dis- 57 ease as feasibly possible to halt said process is a natural incli- 58 nation. Yet, historically, a guiding principle in treating cancer has been that targeting metastatic lesions leads to poor outcomes, because the treatment is either too late or too morbid. However, with advancing imaging technology and increasingly precise techniques for targeting lesions, a much larger proportion of metastatic disease can be treated (7). As a result, the life-extending benefit of targeting metastatic disease is becoming increasingly clear.

Metastatic stage is typically described as a binary variable in a clinical setting, either present or not (M0 or M1), although certain cancer subtypes (e.g. colon, prostate) now have more gradiation in classifying a patient's metastatic stage (8). The term "oligometastatic state" was first described in 1995 as an intermediary between localized and widespread metastatic disease where metastasis-directed treatment has the potential to be curative (9). Since then, results from several exploratory studies and randomized controlled trials using metastasis-directed therapy in such patients have accumulated to support its existence $(10,11)$.

Consensus definitions have since been proposed to further refine subgroups of oligometastasis (12-14). For example, the distinction between oligometastatic disease at presentation versus the development of oligometastatic disease following definitive treatment of non-metastatic cancer have been designated "synchronous oligometastases" and "metachronous oligorecurrence," respectively. "Oligoprogression" describes growth of few metastases in the setting of otherwise stable (or responsive) disease whilst undergoing systemic therapy, and "oligopersistence" is characterized by having several lesions which have a poorer response to systemic therapy than others. Intra-patient heterogeneity often complicates diagnostics even further, where some lesions respond to therapeutics while others persist. These designations (and many more not listed) underscore the complexity with which researchers and clinicians are coming to understand this disease state.

In addition to refining the term "oligometastatic," clinicians have examined the benefit of treating patients with oligometastases $(28,29)$. The implicit null hypothesis of these investigations, that targeting metastatic disease does not provide a life-extending benefit, stems from the current paradigm of metastatic cancer treatment. Table 1 summarizes the results of some of these recent phase II and III clinical trials, demonstrating that this null hypothesis is freNOTE: This preprint reports new research that has not been certified by peer review and should not be used to guide clinical practice. 
It is made available under a CC-BY-NC-ND 4.0 International license .

\begin{tabular}{|c|c|c|c|c|}
\hline Citation & CT Phase & $\begin{array}{l}\text { Primary } \\
\text { Location }\end{array}$ & Results & Description \\
\hline$(15,16)$ & II & NSCLC & $\begin{array}{l}\text { Positive for PFS and } \\
\text { OS }\end{array}$ & $\begin{array}{l}\text { In the Gomez et al. trial, treating oligometastases }(\leq 3 \\
\text { non-primary lesions) demonstrated significant improve- } \\
\text { ment in PFS and OS compared to maintenance therapy }\end{array}$ \\
\hline (17) & II & $\begin{array}{l}\text { NSCLC } \\
\text { (EGFR/ALK } \\
\text { negative) }\end{array}$ & Positive for PFS & $\begin{array}{l}\text { In the trial by Iyengar et al., targeting the primary } \\
\text { with radiotherapy and oligometastases with SBRT fol- } \\
\text { lowed by maintenance chemotherapy provided signifi- } \\
\text { cantly improved progression-free survival compared to } \\
\text { maintenance chemotherapy alone. }\end{array}$ \\
\hline$(18,19)$ & II & Variety & Positive for OS & $\begin{array}{l}\text { In the "SABR-COMET" trial, treating all sites of } \\
\text { oligometastatic cancer with SABR demonstrated sig- } \\
\text { nificantly improved OS compared to standard palliative } \\
\text { treatment. }\end{array}$ \\
\hline (20) & II & $\begin{array}{l}\text { Prostate } \\
\text { (hormone } \\
\text { sensitive) }\end{array}$ & $\begin{array}{l}\text { Positive for composite } \\
\text { of progression metrics }\end{array}$ & $\begin{array}{l}\text { In the "ORIOLE" trial, treating all sites of oligometas- } \\
\text { tates with SABR led to improved outcomes measured by } \\
\text { 6-month rate of progression (by PSA, imaging, symp- } \\
\text { toms, androgen-deprivation therapy initiation, and sur- } \\
\text { vival) when comparing to observation alone. }\end{array}$ \\
\hline (21) & II & Prostate & $\begin{array}{l}\text { Positive for ADT-free } \\
\text { survival }\end{array}$ & $\begin{array}{l}\text { In the "STOMP" trial, in patients with metachronous } \\
\text { oligometastasis, using metastasis-directed therapy } \\
\text { (SBRT or surgery) provided longer ADT-free survival } \\
\text { compared to surveillance alone. }\end{array}$ \\
\hline$(22,23)$ & II & CRC & Positive for OS & $\begin{array}{l}\text { In the "EORTC } 40004 \text { " trial, treating liver metastases } \\
(<10 \text {, no extrahepatic disease) with RFA, systemic treat- } \\
\text { ment, and +/- resection led to long-term OS improve- } \\
\text { ment compared to systemic treatment alone. }\end{array}$ \\
\hline (24) & II & ES-SCLC & $\begin{array}{l}\text { Positive for PFS, neg- } \\
\text { ative for OS }\end{array}$ & $\begin{array}{l}\text { In the "RTOG 0937" trial, treating oligometastases with } \\
\text { PCI and consolidative radiotherapy to both the chest and } \\
\text { metastases did not improve OS and did delay progres- } \\
\text { sion, compared to PCI alone. }\end{array}$ \\
\hline (25) & III & Prostate & $\begin{array}{l}\text { Positive for PSA pro- } \\
\text { gression, negative for } \\
\text { OS }\end{array}$ & $\begin{array}{l}\text { In the "HORRAD" trial, in patients with metastases to } \\
\text { the bone (any amount), providing radiotherapy to the } \\
\text { prostate along with ADT did not improve OS and did } \\
\text { improve time to PSA progression, compared to ADT } \\
\text { alone. Exploratory subgroup analysis suggested patients } \\
\text { with } \leq 4 \text { bone metastases may benefit from prostate ra- } \\
\text { diotherapy. }\end{array}$ \\
\hline (26) & III & Prostate & $\begin{array}{l}\text { Negative for OS in } \\
\text { complete group, posi- } \\
\text { tive for OS in patients } \\
\text { with lower metastatic } \\
\text { burden }\end{array}$ & $\begin{array}{l}\text { In Arm H of the "STAMPEDE" trial, radiotherapy to } \\
\text { the prostate did not improve OS in unfiltered cohort of } \\
\text { patients, compared to lifelong ADT. However, in a pre- } \\
\text { specified subgroup analysis, significant OS improve- } \\
\text { ment was observed among those with lower metastatic } \\
\text { burden. }\end{array}$ \\
\hline (27) & III & Nasopharynx & $\begin{array}{l}\text { Positive for PFS and } \\
\text { OS }\end{array}$ & $\begin{array}{l}\text { In a trial by You et al., the addition of locoregional radio- } \\
\text { therapy to the primary improved OS and PFS compared } \\
\text { to chemotherapy alone in patients with (oligo- and poly- } \\
\text { ) metastatic nasopharyngeal carcinoma. }\end{array}$ \\
\hline
\end{tabular}

Table 1. A summary of clinical trials that examine the benefit of providing local treatment to patients with oligometastases.

quently (but not always) refuted. Even accounting for known 65 positive publication bias $(30,31)$, there is substantial evi- 66 dence that supports a changing paradigm in the treatment ${ }_{67}$ of oligometastatic patients. However, despite many studies 68 showing a significant increase in overall survival (OS) when 69 metastatic lesions are targeted, the null hypothesis in ongoing 70 clinical trial planning has not changed.

In this work, we suggest shifting this qualitative null hypothesis, and describe a mathematical model which can be used to frame a new, quantitative null. We begin with a very simple formulation of tumor growth, an exponential function, and use it to show that while any amount of cell kill 
will extend survival, in many cases the extent is so small as 124 to be unnoticeable in a clinical context or out-weighed by 125 factors related to toxicity and treatment time. Recasting the ${ }_{226}$ null in these quantitative terms will allow trialists to design ${ }_{127}$ trials specifically to increase understanding of what circum- 128 stances (patient selection, disease burden, tumor growth ki- 129 netics) can lead to improved OS when targeting metastatic ${ }_{130}$ lesions, rather than determining whether targeting metastases 131 can extend survival for patients with (oligo-)metastatic dis- 132 ease. We purposely begin with the most simplistic possible ${ }_{133}$ mathematical model, considering only total disease burden ${ }_{134}$ and doubling time. We do not consider complexities such as 135 space, metastatic locations/connectedness (32), immune in- 136 teractions or any heterogeneities - all things which could be ${ }_{137}$ considered in future iterations, but which make the model less ${ }_{138}$ generalizable. Finally, a sensitivity analysis is performed to 139 confirm that our findings are consistent using alternative ordi- 140 nary differential equations (ODEs) that may be used to model ${ }_{141}$ tumor growth (33).

Due to its breadth, the current qualitative null hypothesis ${ }^{143}$ may be incorrectly accepted or rejected without a quantita- 144 tive model to help design optimal patient and treatment pa- 145 rameters. Numerous qualitative and quantitative prognostic 146 factors exist to help identify patients with metastatic disease 147 which is likely to follow a relatively indolent course. For ${ }^{48}$ example, with slower disease progression, patients are more 149 likely to derive greater benefit from aggressively targeting 150 their metastases. Other characteristics include the number 151 of lesions and organs involved, the time course of presen- 152 tation and progression, tumor histology, patient innate and 153 adaptive immunity, and various biological features (34). It 154 is crucial that we parse through which of these patient char- 155 acteristics can meaningfully affect treatment outcomes in the 156 setting of oligometastasis. By rethinking the null hypothe- 157 sis of metastatic cancer treatment, research efforts can better 158 serve our patients by bringing a deeper understanding of how 159 well treatment works, who it works best for, and when it is 160 most efficacious, rather than continually testing the implicit 161 null hypothesis.

\section{Model}

Modeling Tumor Growth using an Exponential Function. Beginning with a very simple model of tumor growth, an exponential function, we will explore the effect of treatment in scenarios with different growth rates, treatment effectiveness, and timing of the intervention. While this overlooks many of the realities of real human cancers, such as spatial, intra- and inter-tumoral (35-37) heterogeneity, it ${ }_{169}$ captures many of the essential aspects of growth (38). Fur- 170 thermore, in the absence of other specific knowledge, general ${ }_{171}$ arguments can be expounded upon, but additional undeter- ${ }_{172}$ mined complexities can severely limit generalizability. Let ${ }_{173}$ us then model a tumor of size (cell number), $N$, beginning ${ }_{174}$ with a single cell, and a growth rate, $r$, as follows:

$$
N(t)=e^{r t} .
$$

A growth curve built using Equation 1 is displayed in Figure 1 as the black line, denoted "Untreated." The threshold tumor burden (an arbitrary number of $N=100$ for illustrative purposes) which leads to patient death, $N_{T}$, is represented by the horizontal black dashed line in Figure 1. Next, we will assume that a given intervention (e.g. stereotactic body radiation therapy (SBRT), or metastasectomy) is given at some time (e.g. upon detection of a metastasis). The total tumor burden at the time of this treatment is denoted as $N_{d}$ and the number of cells killed is denoted as $N_{c}$ cells; note, this requires $0 \leq N_{c} \leq N_{d}$.

To illustrate how the same intervention (removing $N_{c}$ cells from the tumor) at different times effects our measure of survival, we plot several growth curves together in Figure 1. The time when each of these curves reaches $N_{T}$ is the time of death $\left(t_{d, x}\right)$. The difference $(\Delta t)$ between the unperturbed time of death $\left(t_{d, 1}\right)$ and each subsequent example intervention (e.g. $\Delta t=t_{d, 2}-t_{d, 1}$ ) is the increase in survival. We note that the earlier the intervention occurs (smaller $N_{d}$ ), the greater the $\Delta t$ and, therefore, increase in survival. This is also true if we kill more cells (i.e. $N_{c}$ increases).

While Figure 1 considers how a single intervention will effect the "same" tumor, Supplementary Figure 1 explores the effect of altering tumor growth rate, $r$, on $\Delta t$ after the same intervention. This figure adds a faster tumor growth curve, in addition to the curve seen in Figure 1. The same intervention (removal of $N_{c}$ cells) occurs at the same time points as the slower curve, yet the faster growing tumor has a smaller resulting changes in survival time $(\Delta t f)$ compared to the slower growing tumor $(\Delta t s)$.

Next, we will examine the analytical relationship between the change in survival $(\Delta t)$ to the other parameters $\left(r, N_{c}\right.$, $N_{d}$ ). This requires examining two tumor growth curves, one with unperturbed growth starting at $N_{d}$ and the other with perturbed growth beginning at $\left(N_{d}-N_{c}\right)$. In other words, the perturbed curve will have the same growth characteristics as the unperturbed curve, but it will have $N_{c}$ cells removed as "treatment." Then, we will calculate the offset of time between the two curves when they reach $N_{T}$, i.e. $\Delta t$.

Graphically, we are asking how large the difference on the time axis is between where the treated and untreated curves intersect with $N_{T}$ (the black dashed line), denoted by colored circles in Figure 1 and Supplementary Figure 1. Mathematically, we find the difference between $t_{d, 1}$ and $t_{d, 2}$ : i.e. $\Delta t$ when $\left(N_{d}\right) e^{r t_{1}}=\left(N_{d}-N_{c}\right) e^{r t_{2}}=N_{T}$. This relation is:

$$
\Delta t=\frac{1}{r}\left[\ln \frac{N_{d}}{\left(N_{d}-N_{c}\right)}\right] .
$$

The observations from before are maintained: slower growing tumors (smaller $r$ ), more effective interventions (increasing $N_{c}$ ), and lower burden at time of treatment (lower $N_{d}$ ) make for a larger survival benefit, as we have intuited. Additionally, it is important to note that $\Delta t$ is not dependent on the threshold chosen for $N_{T}$, where tumor burden leads to death.

Given the intuitive nature of these results, one may question what the value of such a model is. First, this model al- 


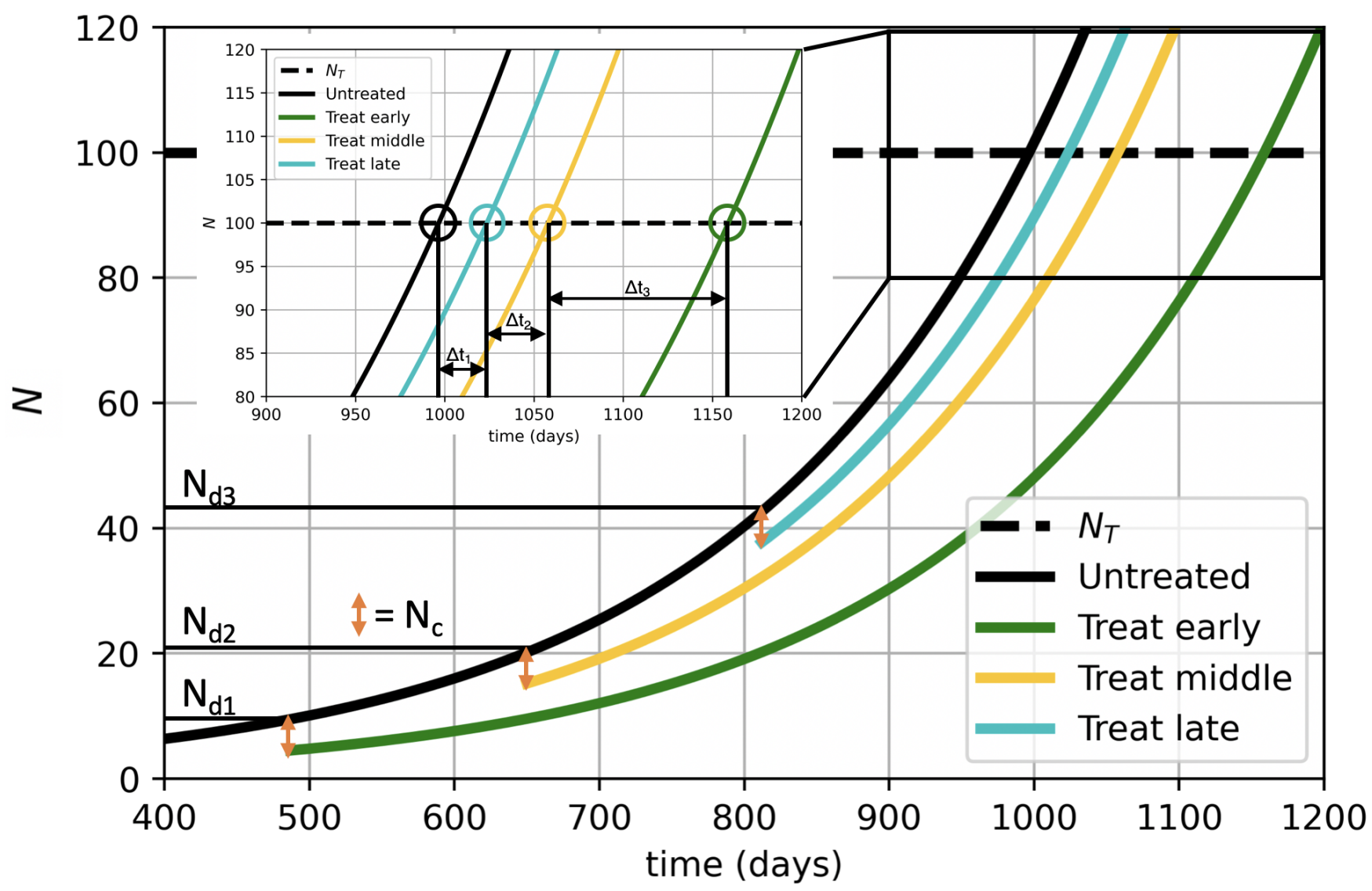

Fig. 1. Change in OS is modulated by when an oligometastasis-directed intervention occurs and the effectiveness of the intervention. We plot an illustrative exponential growth curve from Equation 1 in black. At three different times, we subtract $N_{c}$ cells from the curve to simulate an oligometastasis-directed intervention (orange markers), and the tumor continues to grow at the original rate from the new size. These subsequent tumors then grow and eventually intersect an arbitrary threshold cell (a surrogate for maximum tolerated disease burden) number ( $N_{T}$ - dashed horizontal line), there we can then determine the change in survival (vertical black lines, inset). The change in this time represents the $\Delta t$ for each intervention. n.b. These are not realistic parameters, but instead serve to illustrate the (qualitatively conserved) phenomenon.

lows for the quantitative exploration of what was previously 202 an exclusively qualitatively described phenomenon. This al- 203 lows for formal interrogation of the individual values of each 204 parameter, a crucial step in quantitative reasoning during 205 clinical trial design. In doing so, a framework for parame- 206 ter estimation can help trialists perform sensible power cal- 207 culations. This would require measuring distributions of each 208 of these parameters as it is clear that heterogeneity (and un- 209 certainty) exists in each. Further, this would allow for error 210 propagation in addition to power calculations. With recent ${ }_{211}$ work trying to incorporate toxicity into survival analyses in 212 radiation oncology (39), we have the opportunity to formally ${ }_{213}$ probe the balance between benefit and harm in this setting. 214 Most importantly however, it will remove the confusion created when we test a qualitative null that is likely neither able 215 to be rejected or upheld given the sensitivity to the noise in- 216 herent in clinical data.

Sensitivity Analysis with Alternative Tumor Growth Models. In order to assess the consistency of our findings, 220 we examined the seven ODE models used in Murphy et al., 221 2016 (33), where they fit each model using 14 time points 222 from a naked mouse xenograft experiment by Worschech et 223 al., 2009 (40). The models included in this analysis are 224 as follows: exponential, Figure 2A shows all seven models 225 (including the exponential model) built using the parameters denoted by Murphy et al (33). Using these models, Figure $2 \mathrm{~B}$ denotes the $\Delta t$, or change in OS, between the untreated growth curves and growth curves with the same intervention at early, middle, and late timing. Here, we see that all models show that the same intervention has a greater benefit the earlier it is performed. Each model with untreated, early, middle, and late interventions is individually plotted in Supplementary Figure 2B-H, while Supplementary Figure $2 \mathrm{~A}$ shows all untreated models together for comparison. Although not all models have as extreme of a difference as seen in the exponential model, this quantitative trend, which is the main thrust of this study, remains consistent.

Parameter Sweep of Exponential Growth Model. Figure 3 demonstrates a benefit of using a quantitative model with a sensitivity analysis to help us better understand the areas of the (very simplified) parameter space, a range of possible parameter values, where the greatest opportunities lie. Given that this is a simple exponential relation, the change in survival is monotone (always up or down) in each parameter. However, as the tumor growth curves are non-linear, we chose to plot the sensitivity analysis on a log-log plane to improve the visualization of changes in parameter values.

As we do not currently have known values for these pa- 


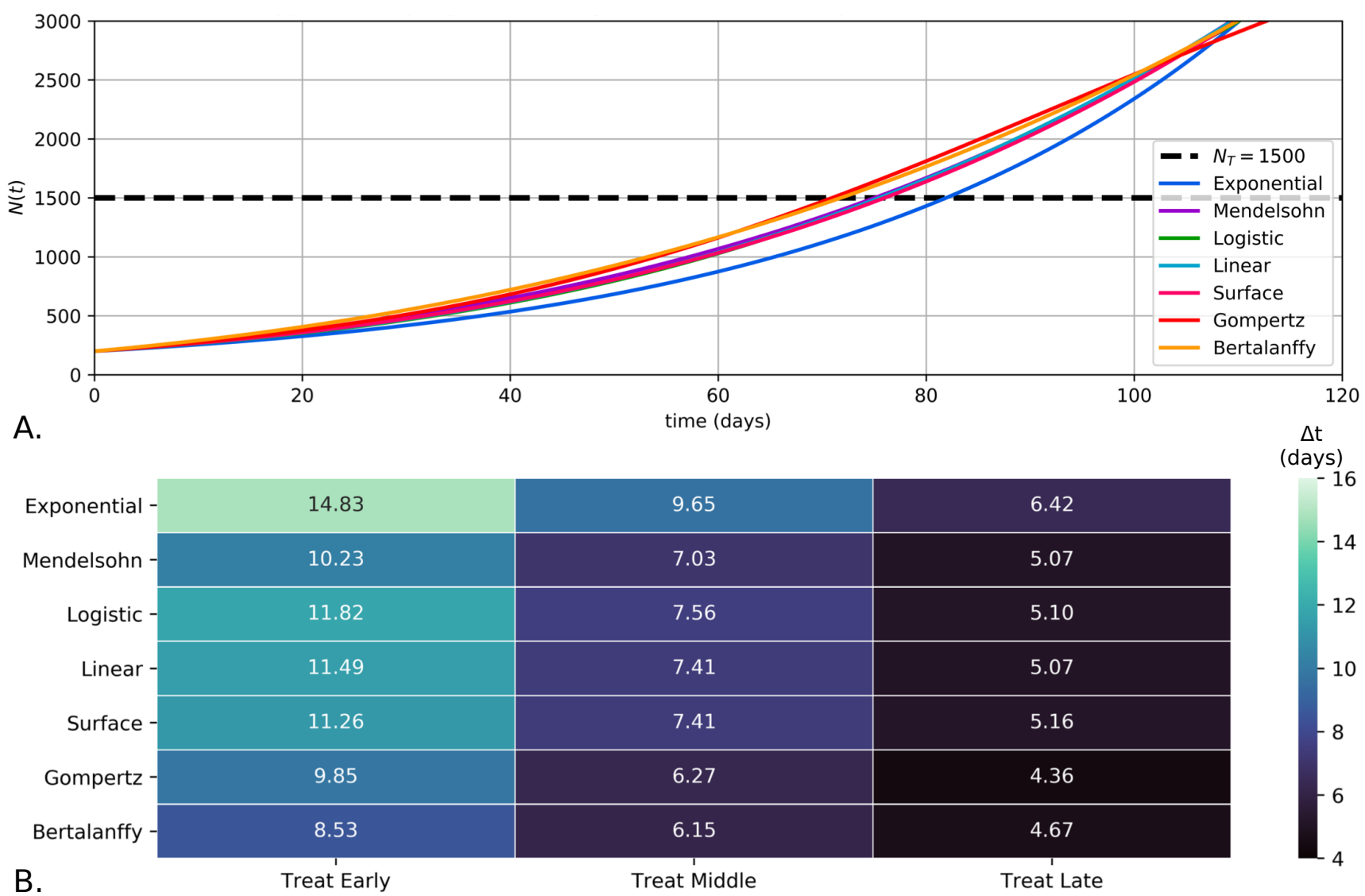

Fig. 2. Across seven ODE tumor growth models, earlier intervention creates a larger improvement in OS. Models were produced using the parameters denoted by Murphy et al (33), where the seven models were fit to 14 timepoints of xenograft tumor growth data from Worschech et al (40). A. A comparison of the seven growth curves with no interventions built with various ODE models. Individual plots for each model and three intervention time points may be found in Supplementary Figure 2. B. A heatmap demonstrating the change in OS ( $\Delta t$ days) for the same intervention $\left(N_{C}=100\right)$ at three different time points for each ODE model. Each heatmap entry is annotated with the exact change in OS for the given model and intervention timing. Treat early, treat middle, and treat late denote the intervention occurring at 20,35 , and 50 days, respectively.

rameters, exploring a large sweep of values can be instruc- 250 tive. We consider a continuous range for $N_{c}$ in $\left[0, N_{d}\right],{ }_{251}$ where $N_{c}=0$ represents no intervention and $N_{c}=N_{d}$ repre- 252 sents a cure. In these cases, $\Delta t=0$ and $\Delta t=\infty$, respec- 253 tively. In Figure 3, we will consider four discrete exam- 254 ples of values for $r$, as this parameter's effect is monotone (where a case with lower $r$ always derives more benefit from ${ }_{255}$ oligometastasis-directed therapy than a case with higher $r$ ). ${ }_{256}$ It is also important to note that this parameter is likely modi- ${ }^{257}$ fiable with chemo- or targeted-therapy: something we do not ${ }_{258}$ consider here, but would be a straightforward extension. This ${ }_{259}$ example will consider growth rates which correspond to tu- ${ }_{260}$ mor doubling times of 100,200, 300 and 400 days. These ${ }_{261}$ could represent tumors such as small cell lung cancer in the ${ }_{262}$ fast extreme or prostate cancer in the slow extreme. Figure $3_{263}{ }_{262}$ shows this analysis, with isoclines shown in black to denote ${ }_{264}$ lines of equal effect. These curves demonstrate that any in- ${ }^{265}$ crease in $N_{c}$ (more cell kill per intervention, "up" on the yaxis) and/or decrease in $N_{d}$ (earlier intervention, "down" on ${ }_{267}$ the x-axis) increases the OS benefit. It is interesting to note ${ }_{268}$ that the movements (i.e. $N_{c}$ up and $N_{d}$ down) mirror the his- ${ }_{269}$ torical trend: improvements in detection of oligometastasis ${ }_{270}$ via anatomic or functional imaging have slowly pushed $N_{d_{271}}$ lower over the years and the ability to safely (using SBRT 272 or minimally invasive surgery with continually lowering toxicity) target larger and larger lesions (increasing $N_{c}$ ) has increased. This "creep" of these values is one reason why the need for a recasting of the null hypothesis is becoming clear, and why the null was historically of greater clinical utility.

\section{Sample Size Calculation Informed by Exponential}

Growth Model. Finally, we performed theoretical sample size calculations (using the Sample Size Estimator from Wang et al., 2020 (41)) in four clinical scenarios: a fast growing tumor type which is detected early, a fast growing tumor type which is detected late, a slow growing tumor type which is detected early, and a slow growing tumor type which is growing late. Using Equation 2, we assume parameters which are congruent with each clinical scenario and calculate the change in survival time between the treated and untreated growth curves. These $\Delta t$ 's are then converted into hazard ratios (HR) relative to each other, with the largest change in survival time relating to the most extreme HR $(0.50)$ and the smallest change in survival time leading to the HR closest to $1(0.80)$. In Table 2, we see that as the HR approaches 1, the predicted sample size requirements become unreasonable for any oncology treatment clinical trial. This HR may occur in a trial with a fast growing tumor type (e.g. SCLC) that has 

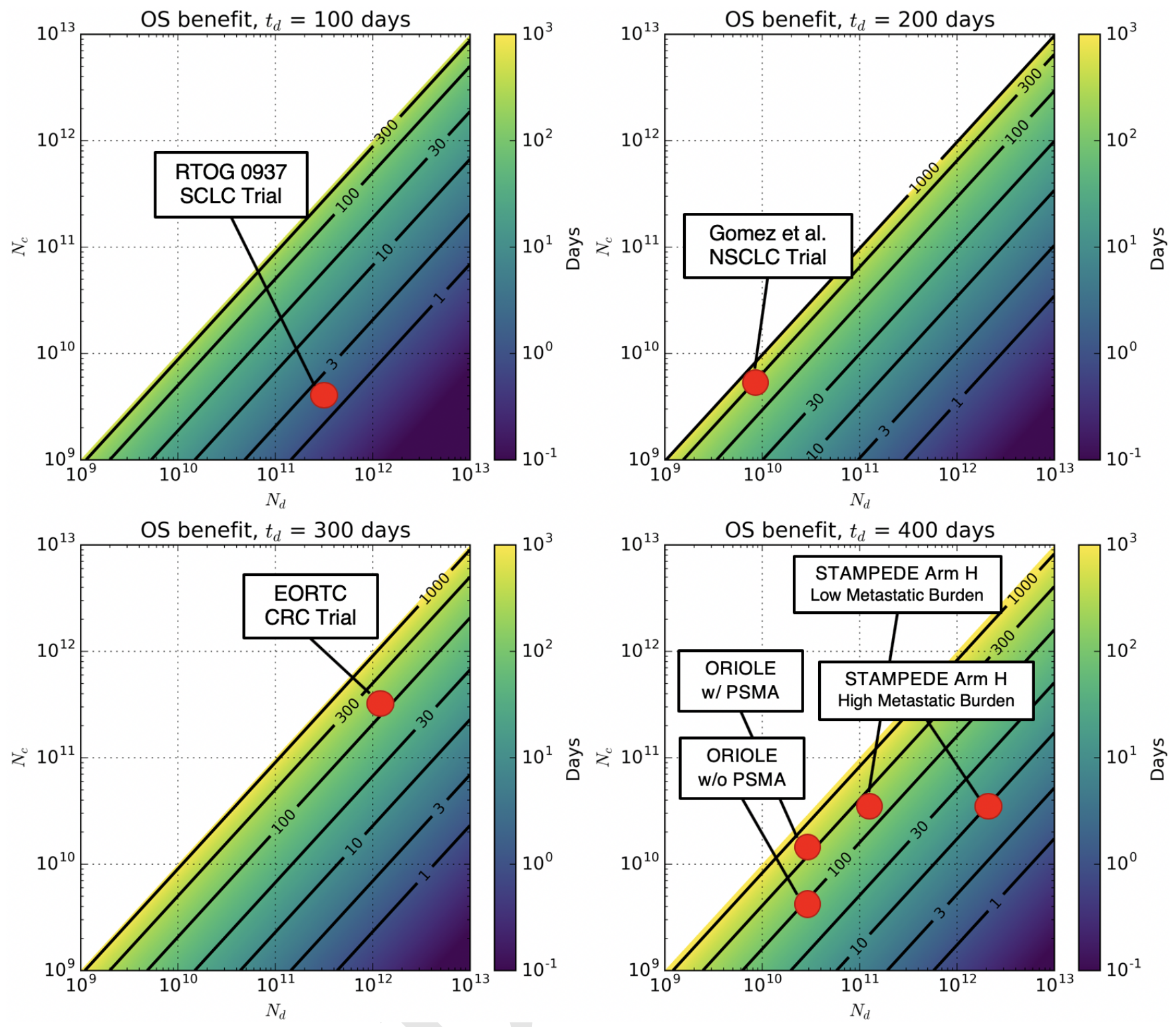

Fig. 3. The benefit of oligometastasis-directed therapy depends monotonically on the amount of cells killed, the tumor burden, and tumor doubling time. We plot four orders of magnitude of both $N_{c}$ and $N_{d}$ on a log scale. The color represents the predicted number of days of OS (PFS in the ORIOLE trial) benefit for each combination of $N_{c}$ and $N_{d}$. Each of the four subplots represents a different "intrinsic" biology, modeled by different tumor doubling times. A $t_{d}$ of $100,200,300$, and 400 days corresponds to a growth rate, $r$, of $0.0069,0.0035,0.0023$, and 0.0017 , respectively. Contour lines are shown for ease of comparison. A selection of trials from Table 1 are represented by red circles based on estimations of $N_{d}, N_{c}, r$, and $t_{d}$ for each trial.

\begin{tabular}{llllllll}
\hline Tumor Parameters & $\begin{array}{l}\text { Doubling } \\
\text { Time (days) }\end{array}$ & $\begin{array}{l}\text { Growth } \\
\text { Rate }(r)\end{array}$ & $N_{d}$ & $N_{c}$ & $\Delta t$ (days) & HR & $\begin{array}{l}\text { Sample } \\
\text { Size }\end{array}$ \\
\hline $\begin{array}{l}\text { Fast growing tumor, early } \\
\text { detection }\end{array}$ & 100 & 0.0069 & $10 E 10$ & $5 E 10$ & 100.0 & 0.60 & 82 \\
\hline $\begin{array}{l}\text { Fast growing tumor, late } \\
\text { detection }\end{array}$ & 100 & 0.0069 & $10 E 11$ & $5 E 10$ & 7.4 & 0.80 & 14698 \\
\hline $\begin{array}{l}\text { Slow growing tumor, } \\
\text { early detection }\end{array}$ & 400 & 0.0017 & $10 E 10$ & $5 E 10$ & 400.0 & 0.50 & 34 \\
\hline $\begin{array}{l}\text { Slow growing tumor, late } \\
\text { detection }\end{array}$ & 400 & 0.0017 & $10 E 11$ & $5 E 10$ & 29.6 & 0.70 & 322 \\
\hline
\end{tabular}

Table 2. Theoretical sample size calculations demonstrate that as the change in OS decreases, the hazard ratio (HR) gets closer to 1, and a larger sample size is predicted. Using Equation 2, $\Delta t$, representing change in OS, was calculated for four clinical scenarios. This predicted change in OS is translated to an estimated hazard ratio to perform a sample size calculation. The sample size calculations assumed a type I error $(\alpha)$ of 0.05 , power $(1-\beta)$ of 0.80 , equal size of treatment arms, and a

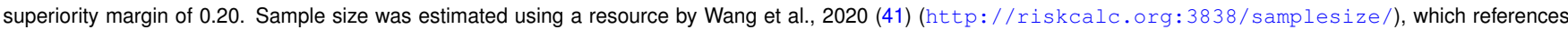
Schoenfeld et al., 1981 (42) and 1983 (43) for the calculation of sample size in parallel randomized control trials for assessing superiority of a treatment using time-to-event. 
widespread metastatic disease.

As seen in McClatchy III et al., 2020 (44) and XXXX et 330 al., 2020 (39), a more technical method of assessing these ${ }_{331}$ theoretical sample size calculations would be to perform an ${ }_{332}$ in silico clinical triald. One would assume distributions for $3 з 3$ each parameter of interest (e.g. $r, N_{d}$ ), sample each "patient" ${ }_{334}$ in the trial from these distributions, assess survival time for 335 treated and untreated groups, and calculate the HR between ${ }_{336}$ the two groups. Even with this theoretical approach, how- 337 ever, additional measurements would be required to appro- 338 priately estimate the distributions of the model parameters. Post-trial data publication could provide a wealth of informa- 340 tion to estimate these parameters. For example, tumor imag- 341 ing both before and after treatment could assess $N_{d}$ and $N_{c}$, ${ }_{342}$ while serial tumor biopsies could be used to inform a distri- ${ }_{343}$ bution for tumor growth rate. Without these data, however, ${ }_{344}$ we believe that the simpler method of converting $\Delta t$ to $\mathrm{HR}_{345}$ shown in Table 2, provides an easier-to-interpret example of ${ }_{346}$ sample size calculations.

\section{Clinical correlation}

In order to demonstrate how clinical trial design can ex- ${ }^{350}$ plore the parameter space of this tumor growth model, we ${ }^{351}$ will review some recent clinical trials, which are also listed ${ }^{352}$ in Table 1. This discussion reviews illustrative examples, and ${ }^{353}$ is not an exhaustive list of all clinical trials which test the ben- ${ }^{354}$ efit of targeting oligometastases. For many trials, we will es- ${ }^{355}$ timate where design falls in the parameter space of Figure 3, 356 and discuss how trial design can test the effects of altering ${ }^{357}$ one or more parameters (i.e. $N_{d} N_{c}$, or $r$ ).

In a phase II trial by Gomez et al., 49 patients with ${ }^{359}$ oligometastatic ( $\leq 3$ metastases) non-small cell lung cancer ${ }^{360}$ (NSCLC) without progression after first-line systemic ther- ${ }^{361}$ apy were randomized to either maintenance systemic ther- ${ }^{362}$ apy/surveillance or local consolidative therapy (LCT) to all ${ }^{363}$ sites of residual disease via surgery or radiotherapy. After ${ }^{364}$ interim analysis demonstrated a substantial PFS benefit with ${ }^{365}$ LCT, the trial was closed early and allowed for crossover to ${ }^{366}$ the LCT arm (15). With additional follow up, and despite ${ }^{367}$ crossover, LCT was associated with improved OS of $41.2^{368}$ months vs 17.0 months (16). We placed this trial in the top ${ }^{369}$ right subplot of Figure 3, due to the relatively fast growth ${ }^{370}$ rate of NSCLC, minimal tumor burden ( $\leq 3$ metastases), and ${ }^{371}$ large $N_{c}$ using radiotherapy or surgery.

The SABR-COMET study was a screening phase II trial ${ }_{373}$ which randomized 99 patients with oligometastatic disease ${ }_{374}$ $(\leq 5$ metastases) of various histologies with a controlled 375 primary site to standard palliative therapy with or without 376 stereotactic ablative radiotherapy (SABR) to all metastatic ${ }_{377}$ lesions. The primary endpoint was OS, which was initially ${ }_{378}$ improved with the addition of SABR from 28 months to $48{ }_{379}$ months (19). With additional follow up, results were even 380 more substantial with a median OS of 50 months using SABR 381 versus 28 months in the control arm (45). As this trial in- 382 cludes tumors of many histologies, we cannot place the pos- 383 itive results in a single subplot of Figure 3, but doing so 384 post-hoc patient by patient would be illustrative. The SABR- 385
COMET trial also utilized stratified randomization to ensure that strata of patients with 1-3 metastases and 4-5 metastases were balanced in treatment assignments. Stratified randomization helps balance treatment arm assignments between patient populations with known prognostic factors, and can reduce the risk of type I and II errors in trials with smaller sample sizes ( $<400$ patients) (46). In relation to our model, by creating strata of the number of metastases, the SABRCOMET trial balanced based on tumor burden at the time of treatment $\left(N_{d}\right)$. In future, other trials may consider stratifying based on tumor growth rate (either inferred by tumor type or measured from serial tumor biopsies), the sensitivity of imaging techniques $\left(N_{d}\right.$ or $\left.N_{c}\right)$, or the efficacy of two treatment types $\left(N_{c}\right)$.

In the phase II EORTC 40004 trial, 119 patients with fewer than 10 unresectable colorectal liver metastases and no extrahepatic disease were randomized to systemic therapy with or without local therapy using RFA (with or without resection). Although the primary endpoint of 30 month OS was not met, longer follow up led to improved OS with RFA from 40.5 months to 45.6 months (23). With a relatively slow growing tumor sub-type, a large $N_{d}$, and a moderate OS benefit, we estimated this clinical trial to fall in the bottom left subplot of the model's parameter space found in Figure 3.

The largest study was Arm H of the STAMPEDE trial, which was a phase III trial of 2061 patients with metastatic prostate cancer randomized to androgen deprivation therapy with or without definitive radiotherapy to the prostate. Prespecified subgroup analysis demonstrated no benefit to the addition of prostate radiotherapy among those with a high metastatic burden, defined as either visceral metastases or $\geq 4$ bone metastases with $\geq 1$ outside of the vertebral bodies or pelvis. However, in the group of 819 patients with a low metastatic burden, radiotherapy to the prostate improved three-year OS from 73 percent to $81 \%$. (26) In relation to our model, this is equivalent to assuming that the two groups (high and low metastatic burden) have different $N_{d}$ at the time of treatment, but experience the same $N_{c}$. It should be noted that unlike other trials discussed, local therapy was delivered only to the primary site, but not the metastatic sites, suggesting a benefit to cytoreduction. The estimated parameter space position of these two subgroups (high metastatic burden and low metastatic burden) is found in the bottom right subplot of Figure 3.

In the ORIOLE trial, patients with metachronous oligometastatic prostate cancer with $\leq 3$ sites as detected by conventional imaging were randomized to surveillance or SABR to all sites (20). The primary endpoint was a composite of disease progression metrics at 6 months, which was improved with SABR at $19 \%$ versus $61 \%$ in the control arm. Interestingly, a subgroup of patients underwent advanced imaging with PSMA PET, which has demonstrated greater sensitivity in detecting prostate cancer metastases (putatively lowering $N_{d}$ ) (47). Among those patients where all PSMA PET avid sites were treated, the 6 month progression rate was just $5 \%$ compared to $38 \%$ in those with untreated sites. This subgroup analysis further supports that advanced imaging can 
better identify metastases and treating all sites improves out- 442 comes. By utilizing a more sensitive technology in detecting ${ }_{443}$ (and therefore targeting) metastases, we see that a greater $N_{c 44}$ increases PFS, even if $N_{d}$ remains the same. We estimate ${ }_{445}$ the parameter space for this subgroup analysis in the bottom ${ }_{446}$ right subplot of Figure 3.

Not all trials have demonstrated benefit to the addition of ${ }_{448}$ metastasis-directed therapy. For example, RTOG 0937 was a 449 phase II study of 86 patients with extensive stage small cell 450 lung cancer with at least a partial response to chemotherapy 451 and 1-4 extracranial metastases who were randomized to pro- 452 phylactic cranial irradiation with or without consolidative ra- ${ }^{453}$ diotherapy to the chest and all metastatic sites. The primary ${ }_{454}$ endpoint of one-year OS was not significantly different; $60 \% 455$ in the control arm and $51 \%$ in the consolidative radiotherapy 456 arm (24). This negative result is estimated to be in the top left ${ }_{457}$ subplot of Figure 3, due to the rapid growth of SCLC. Here, 458 this model could have still been useful by informing the sam- 459 ple size calculations (Table 2), given the parameter estimates 460 of the trial's patient population.

\section{Conclusions}

In this work, we have used a simple exponential model ${ }_{465}^{464}$ of tumor growth to demonstrate why recent improvements in ${ }_{466}$ metastasis detection and treatment may allow us to reconsider ${ }_{467}$ the null hypothesis when treating patients with oligometas- ${ }_{468}$ tases. Specifically, more sensitive techniques to localize ${ }_{469}$ metastases (as seen with PSMA imaging) increases how many tumor cells are removed, $N_{c}$, when considering patients at similar stages. When used for surveillance, these ${ }^{470}$ imaging techniques can decrease the tumor burden at the time ${ }_{471}$ of treatment, $N_{d}$, while still increasing the efficacy of ther- 472 apy, $N_{c}$, potentially leading to clinically significant improved OS. Next, advancements in the ability to administer local therapy to all sites of disease with surgical resection, radiotherapy, and/or ablative procedures such as radiofrequency 475 ablation (RFA) has allowed for more effective, precise eradi- ${ }_{477}^{476}$ cation of metastatic lesions with reduced associated morbid- 478 ity. Furthermore, novel immuno- and targeted-therapies can ${ }^{479}$ likely decrease the growth rate, $r$, of tumors.

A mathematical model provides the distinct advantage of ${ }_{480}$ testing quantitative hypotheses to optimize the treatment of patients with oligometastases. Parameter selection regard- ${ }_{482}$ ing number of oligometastases, measurements of tumor bur- ${ }^{483}$ den, and efficacy of treatment options can be examined with ${ }^{485}$ robust hypotheses born from simulated results. Addition- ${ }_{487}^{486}$ ally, with increased translation between the bench and bed- ${ }^{488}$ side, some model parameters (e.g. $r$, tumor growth rate) ${ }_{490}^{489}$ may be inferred using serial tumor biopsies, in vitro, or in ${ }_{491}$ silico modeling. A deeper understanding of how these pa- ${ }_{493}^{492}$ rameters affect outcome can improve trial design by allow- 494 ing for rational prognostic strata criteria in stratified random- ${ }^{495}$ ization or informing prior probabilities in a Bayesian clin- ${ }^{496}$ ical trial $(46,48,49)$. Furthermore, Bayesian trial interim ${ }_{499}^{498}$ analyses can be enhanced with additional simulations using ${ }_{500}^{499}$ updated parameters as patient characteristics are observed or ${ }^{501}$ assessed over time. With a better understanding of the prog- ${ }^{503}$ nostic factors of the population enrolled in a trial, these interim analyses may be used to update prior probabilities, predict probability of success, and assess sample size requirements $(48,49)$.

It is important to note that the model demonstrated in this work is not a perfect representation of tumor growth and treatment, as it fails to consider intratumoral heterogeneity, metastasis location, and the inherent risks of treatment. However, because of its simplicity, this model provides a foundation exploring the current parameter space, while allowing researchers to add complexity as they see fit.

There are minimal published clinical trial results which support upholding the current paradigm in the treatment of oligometastases; however, this is likely due in part to publication bias where positive results are more likely to be published, not simply because this null hypothesis has always been rejected $(30,31)$. The clinical trials examined in this manuscript have necessarily sought to examine the fundamental idea that oligometastatic lesions should only be targeted for palliative care. Refuting this standard was crucial, as the earlier state of cancer imaging and treatment established that targeting oligometastases either occurred too late or caused too much harm. Yet, as quantitative models of tumor growth and the knowledge of how metastatic detection and treatment have evolved, we believe that clinical trials can now provide an even greater benefit by reconsidering the default null hypothesis and utilizing the quantitative principles of this mathematical model in trial design.

\section{Code Availability}

All code used to create mathematical models and figures in this manuscript may be found on GitHub at https://github.com/jessicascarborough/ oligomet-null-hypothesis.

\section{ACKNOWLEDGEMENTS}

JGS thanks his patients for providing him with motivation to push the boundaries of what we know. He would also like to thank the NIH for their support through NIH R37CA244613 and their generous Loan Repayment Program and the American Cancer Society for the Research Scholar Grant (Award number: 132691-RSG-20096-01-CSM). JAS was supported in part by NIH grant T32 GM007250.

\section{Bibliography}

1. Christine $L$ Chaffer and Robert $A$ Weinberg. A perspective on cancer cell metastasis. Science, 331(6024):1559-1564, 2011.

2. Hanna Dillekås, Michael S Rogers, and Oddbjørn Straume. Are $90 \%$ of deaths from cancer caused by metastases? Cancer medicine, 8(12):5574-5576, 2019

3. Thomas N Seyfried and Leanne C Huysentruyt. On the origin of cancer metastasis. Critical reviews in oncogenesis, 18(1-2):43, 2013

4. Douglas Hanahan and Robert A Weinberg. The hallmarks of cancer. cell, 100(1):57-70, 2000.

5. Jacob Scott, Peter Kuhn, and Alexander RA Anderson. Unifying metastasis-integrating intravasation, circulation and end-organ colonization. Nature Reviews Cancer, 12(7):445446, 2012.

6. Scott Valastyan and Robert A Weinberg. Tumor metastasis: molecular insights and evolving paradigms. Cell, 147(2):275-292, 2011.

7. Sondos Zayed, Rohann JM Correa, and David A Palma. Radiation in the treatment of oligometastatic and oligoprogressive disease: Rationale, recent data, and research questions. The Cancer Journal, 26(2):156-165, 2020.

8. Mahul B Amin, Frederick L Greene, Stephen B Edge, Carolyn C Compton, Jeffrey E Gershenwald, Robert K Brookland, Laura Meyer, Donna M Gress, David R Byrd, and David P Winchester. The eighth edition ajcc cancer staging manual: continuing to build a bridge from a population-based to a more "personalized" approach to cancer staging. CA: a cancer journal for clinicians, 67(2):93-99, 2017.

9. Samuel Hellman and Ralph R Weichselbaum. Oligometastases. Journal of Clinical Oncology, 13(1):8-10, 1995. 
10. Sean P Pitroda, Nikolai N Khodarev, Lei Huang, Abhineet Uppal, Sean C Wightman, Sabha 590 Ganai, Nora Joseph, Jason Pitt, Miguel Brown, Martin Forde, et al. Integrated molecu- 591 lar subtyping defines a curable oligometastatic state in colorectal liver metastasis. Nature 592 communications, 9(1):1-8, 2018.

1. Julian C Hong, Diandra N Ayala-Peacock, Jason Lee, A William Blackstock, Paul Okunieff, 594 Max W Sung, Ralph R Weichselbaum, Johnny Kao, James J Urbanic, Michael T Milano, 595 et al. Classification for long-term survival in oligometastatic patients treated with ablative 596 radiotherapy: a multi-institutional pooled analysis. PloS one, 13(4):e0195149, 2018.

12. Ralph R Weichselbaum and Samuel Hellman. Oligometastases revisited. Nature reviews 598 Clinical oncology, 8(6):378, 2011

13. Matthias Guckenberger, Yolande Lievens, Angelique B Bouma, Laurence Collette, Andre 600 Dekker, M deSouza Nandita, Anne-Marie C Dingemans, Beatrice Fournier, Coen Hurk- 601 mans, Frédéric E Lecouvet, et al. Characterisation and classification of oligometastatic 602 disease: a european society for radiotherapy and oncology and european organisation for 603 research and treatment of cancer consensus recommendation. The Lancet Oncology, 21604 (1):e18-e28, 2020

4. Yolande Lievens, Matthias Guckenberger, Daniel Gomez, Morten Hoyer, Puneeth lyengar, 606 Isabelle Kindts, Alejandra Méndez Romero, Daan Nevens, David Palma, Catherine Park, 607 et al. Defining oligometastatic disease from a radiation oncology perspective: An estro- 608 astro consensus document. Radiotherapy and Oncology, 2020.

15. Daniel R Gomez, George R Blumenschein Jr, J Jack Lee, Mike Hernandez, Rong Ye, 610 D Ross Camidge, Robert C Doebele, Ferdinandos Skoulidis, Laurie E Gaspar, Don L Gib- 611 bons, et al. Local consolidative therapy versus maintenance therapy or observation for 612 patients with oligometastatic non-small-cell lung cancer without progression after first-line 613 systemic therapy: a multicentre, randomised, controlled, phase 2 study. The lancet oncol- 614 ogy, 17(12):1672-1682, 2016.

6. Daniel R Gomez, Chad Tang, Jianjun Zhang, George R Blumenschein Jr, Mike Hernandez, 616 J Jack Lee, Rong Ye, David A Palma, Alexander V Louie, D Ross Camidge, et al. Local con- 617 solidative therapy vs. maintenance therapy or observation for patients with oligometastatic 618 non-small-cell lung cancer: long-term results of a multi-institutional, phase ii, randomized 619 study. Journal of Clinical Oncology, 37(18):1558-1565, 2019.

17. Puneeth lyengar, Zabi Wardak, David E Gerber, Vasu Tumati, Chul Ahn, Randall S Hughes, 621 Jonathan E Dowell, Naga Cheedella, Lucien Nedzi, Kenneth D Westover, et al. Consolida- 622 tive radiotherapy for limited metastatic non-small-cell lung cancer: A phase 2 randomized 623 clinical trial. JAMA oncology, 4(1):e173501-e173501, 2018.

18. David A Palma, Cornelis JA Haasbeek, George B Rodrigues, Max Dahele, Michael Lock, 625 Brian Yaremko, Robert Olson, Mitchell Liu, Jason Panarotto, GwendolynHMJ Griffioen, et al. 626 Stereotactic ablative radiotherapy for comprehensive treatment of oligometastatic tumors 627 (sabr-comet): study protocol for a randomized phase ii trial. BMC cancer, 12(1):305, 2012. 628

19. David A Palma, Robert Olson, Stephen Harrow, Stewart Gaede, Alexander V Louie, Cor- 629 nelis Haasbeek, Liam Mulroy, Michael Lock, George B Rodrigues, Brian P Yaremko, et al. 630 Stereotactic ablative radiotherapy versus standard of care palliative treatment in patients 631 with oligometastatic cancers (sabr-comet): a randomised, phase 2, open-label trial. The 632 Lancet, 393(10185):2051-2058, 2019.

20. Ryan Phillips, William Yue Shi, Matthew Deek, Noura Radwan, Su Jin Lim, Emmanuel S 634 Antonarakis, Steven P Rowe, Ashley E Ross, Michael A Gorin, Curtiland Deville, et al. Out- 635 comes of observation vs stereotactic ablative radiation for oligometastatic prostate cancer: 636 the oriole phase 2 randomized clinical trial. JAMA oncology, 6(5):650-659, 2020.

21. Piet Ost, Dries Reynders, Karel Decaestecker, Valérie Fonteyne, Nicolaas Lumen, Aurelie 638 DeBruycker, Bieke Lambert, Louke Delrue, Renée Bultijnck, Tom Claeys, et al. Surveillance 639 or metastasis-directed therapy for oligometastatic prostate cancer recurrence: a prospec- 640 tive, randomized, multicenter phase ii trial. Journal of Clinical Oncology, 2017.

22. T Ruers, C Punt, F Van Coevorden, JPEN Pierie, I Borel-Rinkes, JA Ledermann, G Poston, 642 W Bechstein, MA Lentz, M Mauer, et al. Radiofrequency ablation combined with systemic 643 treatment versus systemic treatment alone in patients with non-resectable colorectal liver 644 metastases: a randomized eortc intergroup phase ii study (eortc 40004). Annals of Oncol- 645 ogy, 23(10):2619-2626, 2012.

23. Theo Ruers, Frits Van Coevorden, Cornelis JA Punt, Jean-Pierre EN Pierie, Inne Borel- 647 Rinkes, Jonathan A Ledermann, Graeme Poston, Wolf Bechstein, Marie-Ange Lentz, 648 Murielle Mauer, et al. Local treatment of unresectable colorectal liver metastases: results of 649 a randomized phase ii trial. JNCI: Journal of the National Cancer Institute, 109(9):djx015, 650 2017.

24. Elizabeth M Gore, Chen Hu, Alexander Y Sun, Daniel F Grimm, Suresh S Ramalingam, Neal E Dunlap, Kristin A Higgins, Maria Werner-Wasik, Aaron M Allen, Puneeth lyengar, et al. Randomized phase ii study comparing prophylactic cranial irradiation alone to prophylactic cranial irradiation and consolidative extracranial irradiation for extensive-disease small cell lung cancer (ed sclc): Nrg oncology rtog 0937. Journal of Thoracic Oncology, 12 (10):1561-1570, 2017

25. Liselotte MS Boevé, Maarten CCM Hulshof, André N Vis, Aeilko H Zwinderman, Jos WR Twisk, Wim PJ Witjes, Karl PJ Delaere, R Jeroen A van Moorselaar, Paul CMS Verhagen, and George van Andel. Effect on survival of androgen deprivation therapy alone compared to androgen deprivation therapy combined with concurrent radiation therapy to the prostate in patients with primary bone metastatic prostate cancer in a prospective randomised clinical trial: data from the horrad trial. European urology, 75(3):410-418, 2019.

26. Christopher C Parker, Nicholas D James, Christopher D Brawley, Noel W Clarke, Alex P Hoyle, Adnan Ali, Alastair WS Ritchie, Gerhardt Attard, Simon Chowdhury, William Cross, et al. Radiotherapy to the primary tumour for newly diagnosed, metastatic prostate cance (stampede): a randomised controlled phase 3 trial. The Lancet, 392(10162):2353-2366, 2018.

27. Rui You, You-Ping Liu, Pei-Yu Huang, Xiong Zou, Rui Sun, Yu-Xiang He, Yi-Shan Wu, GuoPing Shen, Hong-Dan Zhang, Chong-Yang Duan, et al. Efficacy and safety of locoregional radiotherapy with chemotherapy vs chemotherapy alone in de novo metastatic nasopharyngeal carcinoma: A multicenter phase 3 randomized clinical trial. JAMA oncology, 2020.

28. Deepinder Singh, Won Sam Yi, Ralph A Brasacchio, Ann G Muhs, Therese Smudzin, Jacqueline P Williams, Edward Messing, and Paul Okunieff. Is there a favorable subse of patients with prostate cancer who develop oligometastases? International Journal of
Radiation Oncology* Biology* Physics, 58(1):3-10, 2004

29. David A Palma, Joseph K Salama, Simon S Lo, Suresh Senan, Tom Treasure, Ramaswamy Govindan, and Ralph Weichselbaum. The oligometastatic state-separating truth from wishful thinking. Nature reviews Clinical oncology, 11(9):549, 2014.

30. Ana Mlinarić, Martina Horvat, and Vesna Šupak Smolčić. Dealing with the positive publication bias: Why you should really publish your negative results. Biochemia medica: Biochemia medica, 27(3):447-452, 2017.

31. Mohammad Hassan Murad, Haitao Chu, Lifeng Lin, and Zhen Wang. The effect of publication bias magnitude and direction on the certainty in evidence. BMJ evidence-based medicine, 23(3):84-86, 2018.

32. Jacob G Scott, David Basanta, Alexander RA Anderson, and Philip Gerlee. A mathematica model of tumour self-seeding reveals secondary metastatic deposits as drivers of primary tumour growth. Journal of The Royal Society Interface, 10(82):20130011, 2013.

33. Hope Murphy, Hana Jaafari, and Hana M Dobrovolny. Differences in predictions of ode models of tumor growth: a cautionary example. BMC cancer, 16(1):1-10, 2016.

34. Sean P Pitroda and Ralph R Weichselbaum. Integrated molecular and clinical staging defines the spectrum of metastatic cancer. Nature Reviews Clinical Oncology, 16(9):581-588, 2019.

35. Andriy Marusyk and Kornelia Polyak. Tumor heterogeneity: causes and consequences. Biochimica et Biophysica Acta (BBA)-Reviews on Cancer, 1805(1):105-117, 2010.

36. Jacob G Scott, Alexander G Fletcher, Alexander RA Anderson, and Philip K Maini. Spatial metrics of tumour vascular organisation predict radiation efficacy in a computational model. PLoS computational biology, 12(1):e1004712, 2016.

37. Jacob G Scott, Anders Berglund, Michael J Schell, Ivaylo Mihaylov, William J Fulp, Binglin Yue, Eric Welsh, Jimmy J Caudell, Kamran Ahmed, Tobin S Strom, et al. A genome-based model for adjusting radiotherapy dose (gard): a retrospective, cohort-based study. The Lancet Oncology, 18(2):202-211, 2017.

38. Philip Gerlee. The model muddle: in search of tumor growth laws. Cancer research, 73(8): 2407-2411, 2013.

39. Jacob G Scott, Geoffrey Sedor, Michael W Kattan, Jeffrey Peacock, G Daniel Grass, Eric A Mellon, Ram Thapa, Michael Schell, Anthony Waller, Sean Poppen, et al. Optimizing clinical outcome and toxicity in lung cancer using a genomic marker of radiosensitivity. medRxiv, (JTO, accepted), 2020.

40. Andrea Worschech, Nanhai Chen, A Yu Yong, Qian Zhang, Zoltan Pos, Stephanie Weibel, Viktoria Raab, Marianna Sabatino, Alessandro Monaco, Hui Liu, et al. Systemic treatment of xenografts with vaccinia virus glv- $1 \mathrm{~h} 68$ reveals the immunologic facet of oncolytic therapy. BMC genomics, 10(1):301, 2009

41. Xiaofeng Wang and Xinge Ji. Sample size estimation in clinical research: from randomized controlled trials to observational studies. Chest, 158(1):S12-S20, 2020.

42. David Schoenfeld. The asymptotic properties of nonparametric tests for comparing survival distributions. Biometrika, 68(1):316-319, 1981.

43. David A Schoenfeld. Sample-size formula for the proportional-hazards regression model. Biometrics, pages 499-503, 1983.

44. David M McClatchy, Henning Willers, Aaron N Hata, Zofia Piotrowska, Lecia V Sequist, Harald Paganetti, and Clemens Grassberger. Modeling resistance and recurrence patterns of combined targeted-chemoradiotherapy predicts benefit of shorter induction period. Cancer Research, 80(22):5121-5133, 2020.

45. David Palma, Robert Olson, Stephen Harrow, Stewart Gaede, Alexander V Louie, Cornelis Haasbeek, Liam Mulroy, Michael Lock, George Rodrigues, Brian Yaremko, et al. Stereotactic ablative radiotherapy for the comprehensive treatment of oligometastatic cancers: Longterm results of the sabr-comet phase ii randomized trial. medRxiv, 2020.

46. Walter N Kernan, Catherine M Viscoli, Robert W Makuch, Lawrence M Brass, and Ralph Horwitz. Stratified randomization for clinical trials. Journal of clinical epidemiology, 52(1): 19-26, 1999.

47. Finn E von Eyben, Maria Picchio, Rie von Eyben, Handoo Rhee, and Glenn Bauman. 68ga-labeled prostate-specific membrane antigen ligand positron emission tomography/computed tomography for prostate cancer: a systematic review and meta-analysis. European urology focus, 4(5):686-693, 2018.

48. Matthew A Psioda and Joseph G Ibrahim. Bayesian clinical trial design using historical data that inform the treatment effect. Biostatistics, 20(3):400-415, 2019.

49. Donald A Berry. Bayesian clinical trials. Nature reviews Drug discovery, 5(1):27-36, 2006. 
medRxiv preprint doi: https://doi.org/10.1101/2020.08.10.20172098; this version posted December 19, 2020. The copyright holder for this preprint (which was not certified by peer review) is the author/funder, who has granted medRxiv a license to display the preprint in perpetuity.

It is made available under a CC-BY-NC-ND 4.0 International license .

\section{Supplementary Information}

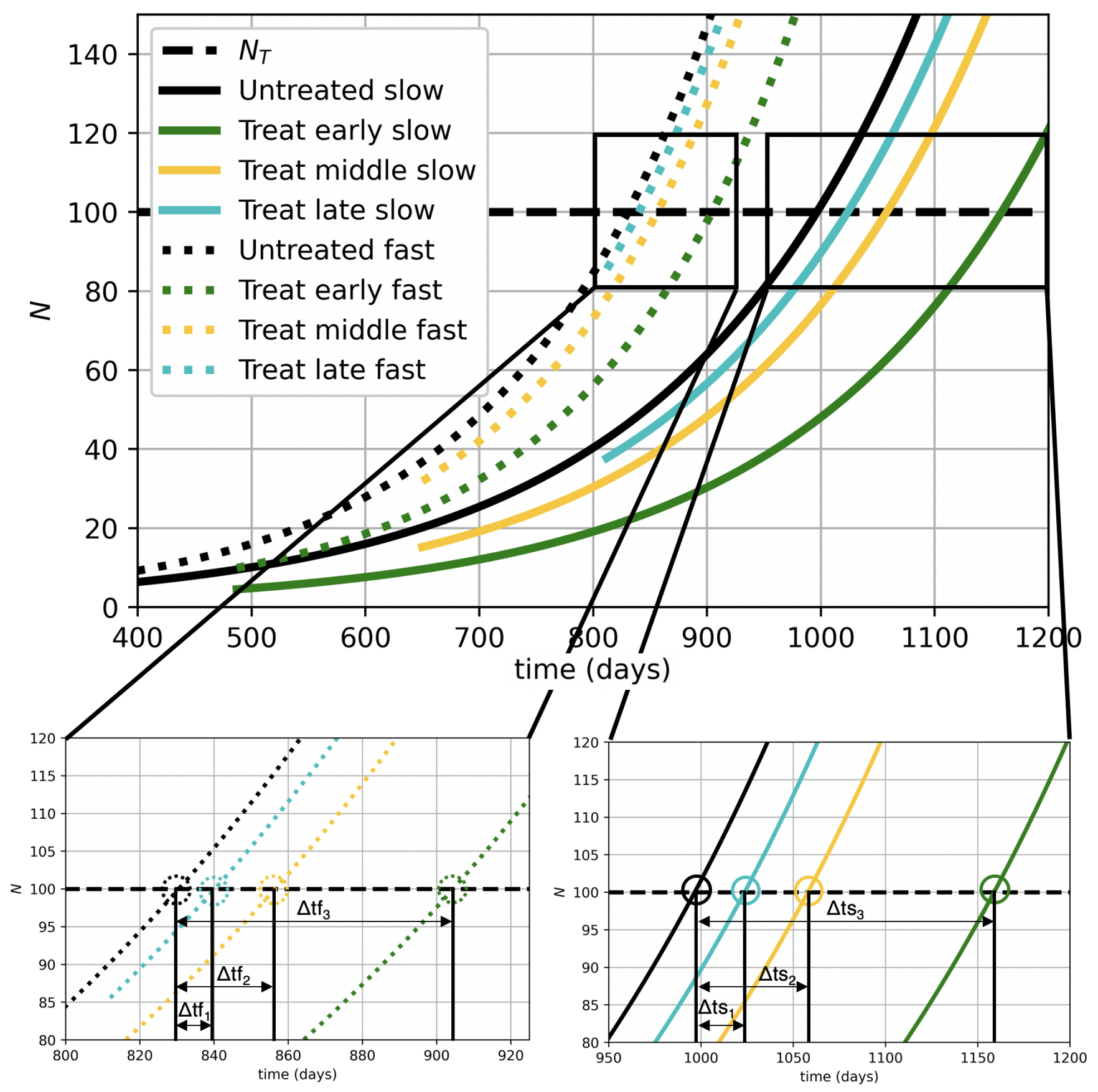

Supplementary Fig 1. Change in OS is modulated by tumor growth rate, intervention timing, and intervention efficacy. Top: We plot two illustrative exponential growth curves from Equation 1 in black, using a faster (dotted line) and slower (solid line) growth rate, $r$. The slower growth rate is the same curves shown in Figure 1. At three different time points, we subtract $N_{c}$ cells from the two curves to simulate an oligometastasis-directed intervention, and the tumor continues to grow at the original rate from the new size. These subsequent tumors then grow and eventually intersect an arbitrary threshold cell (a surrogate for maximum tolerated disease burden) number ( $N_{T}$ - dashed horizontal line). Bottom: We plot two expanded windows of the above plot, showing greater detail of the faster (left, dotted) and slower (right, solid) growth curves as they reach $N_{t}$. In these plots, we can then determine the change in survival (vertical black lines). The change in this time represents the $\Delta t f$ and $\Delta t s$ for each intervention in the fast and slow curves, respectively. Notably, the x-axis for the faster (left, dotted) growth curves accounts for fewer days, despite having the same relative length as the x-axis for the slower (right, solid) growth curves. This was necessary in order to annotate the smaller $\Delta t f$ for the faster growth curves. 
medRxiv preprint doi: https://doi.org/10.1101/2020.08.10.20172098; this version posted December 19, 2020. The copyright holder for this preprint (which was not certified by peer review) is the author/funder, who has granted medRxiv a license to display the preprint in perpetuity.

It is made available under a CC-BY-NC-ND 4.0 International license .
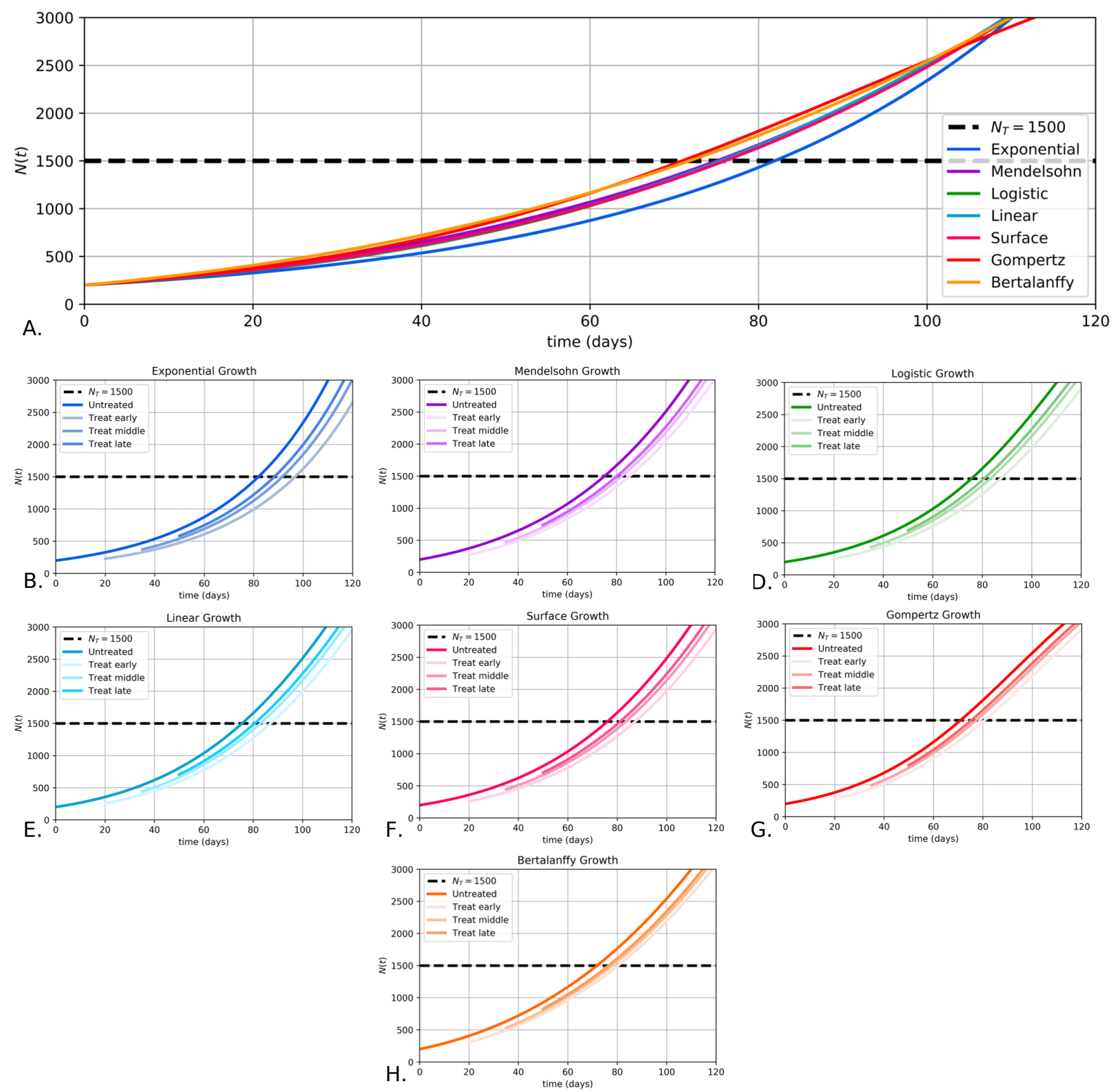

Supplementary Fig 2. A visual representation across seven ODE tumor growth models, demonstrating that earlier intervention creates a larger improvement in OS. Models were produced using the parameters denoted by Murphy et al (33), where the seven models were fit to 14 timepoints of xenograft tumor growth data from Worschech et al (40). A. A comparison of the seven growth curves with no interventions built with various ODE models. B-H. Individual plots visually demonstrating the change in OS for three intervention times for all models included in A. The calculation of change in OS, is demonstrated in Figure 1 with the calculation of $\Delta t$. 